\title{
Microspheres Embedded In Microbeads: A Novel Approach to Improve Various Controlled Release Characteristics of Highly Water Soluble Drug through Ionic Gelation Method
}

\author{
Sunit Kumar Sahoo*, Sabyasachi Swain, Ramakrishna Sen, Deepika Sahoo \\ University Department of Pharmaceutical Sciences, Utkal University, Bhubaneswar, Odisha. India.
}

\begin{abstract}
The major hindrance behind the preparation of gellan microbeads by simple ionic gelation technique with aqueous soluble drugs is their low entrapment efficiency and sometimes its faster dissolution characteristics. This limits the employment of such a simple method for the preparation of microbeads of water-soluble drugs like stavudine. In the present study a novel attempt has been undertaken by embedding stavudine loaded Eudragit RSPO microspheres into gellan microbeads by ionotropic gelation method with an aim to improve its controlled drug delivery characteristics. The prepared microbeads were characterized by scanning electron microscopy, Fourier transform infrared spectroscopy, and optical microscopy for surface topography, drug polymer interaction and particle size analysis respectively. Other studies like drug content, encapsulation efficiency and drug release studies were also carried out. The resulting microspheres embedded in microbeads were free flowing in nature with mean particle size ranging from 544.2-564.2 $\mu \mathrm{m}$. SEM photomicrographs distinctly shows the microspheres embedded within the microbeads effectively with a smooth surface topography and the gellan microbeads containing D4T only are spherical in nature with rough surface. The FTIR study ascertained the compatibility of the drug within the formulation. The microspheres embedded in microbeads had enhanced percentage of drug content, drug entrapment efficiency and controlled release characteristics as compared to beads containing stavudine only. The mechanism of drug release from the formulations were found to follow non Fickian type diffusion with " $n$ " value between 0.5 to 1 but in case of stavudine loaded Eudragit RSPO microspheresit was found to be Fickian type.
\end{abstract}

Key words: Gellan gum, Stavudine, Microbeads, Microsphere embedded in Microbeads, Entrapment efficiency, and Controlled release.

\section{INTRODUCTION}

Controlled release drug delivery systems are designed with a novel idea that will provide optimum bioavailability and therapeutic efficacy while minimizing local and systemic side effects. ${ }^{1-3}$ Ionotropic gelation is one of the most eco-friendly techniques for the encapsulation of the desired drug, as this does not utilise any organic solvent within the formulation. ${ }^{4}$ Naturally occurring Polysaccharides, when cross linked by using various approaches, have shown a great potential for many applications in pharmaceuticals including design of controlled release drug delivery system. ${ }^{4}$ Gellan gum (GG) is an extracellular anionic, hetero polysaccharide produced by Sphingomonas elodea during aerobic fermentation. GG consists of a linear structure of repeating tetrasaccharide units of glucose, glucuronic acid and rhamnose. ${ }^{5,6}$ Gellan is having two forms as high acyl gellan and low acyl gellan. The native polysaccharide (high-acyl gellan) contains approximately 6\% O-acetyl groups, which is lost during alkali treatment of commercial extraction yielding
Submission Date :28-08-2014 Revision Date : :14-10-2014 Accepted Date : :23-12-2014

DOI: 10.5530/ijper.49.2.10 Correspondence Address Dr.S. K. Sahoo University Department of Pharmaceutical Sciences, Utkal University, Bhubaneswar, Odisha. India.

Email:sahoosunitkumar@ gmail.com

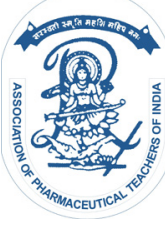

www.ijper.org 
low-acyl GG. This commercial gellan cross-links are more effective with divalent cations than the monovalent cations. ${ }^{5,6}$

The drug of choice for the present study is a water-soluble drug that is stavudine (D4T). The drug (D4T, thymidine) is an FDA-approved drug for clinical use in the treatment of HIV infection, AIDS, and AIDS-related conditions. ${ }^{7}$ According to Bio-pharmaceutics Classification System (BCS), D4T can be assigned to BCS class I. ${ }^{7} \mathrm{D} 4 \mathrm{~T}$ is typically administered orally as a capsule and an oral solution. The virustatic drug has a very short half-life (1.30 h).

Although the process of ionic gelation technique sounds to be a very effective method for designing controlled release microbeads for oral delivery of drugs, but in case of the water soluble drugs the process fails to provide higher percentage encapsulation and improved controlled release characteristics for several reasons.

In the present study, microspheres embedded in microbeads (MEM) were prepared by encapsulating D4T in Eudragit RSPO (ERSPO) microspheres by solvent evaporation technique followed by embedding the same into gellan beads by ionotropic gelation method with an aim to improve its controlled drug delivery characteristics in comparison to beads prepared by simple ionic gelation technique containing D4T only.

\section{MATERIALS AND METHODS}

\section{Materials}

Deacetylated Gellan gum (GG), Kelcogel囚, was obtained as a gift sample from CP Kelco (Burzin and Leons, Pvt. Ltd.), Mumbai (India). D4T and ERSPO were obtained as gift samples from Hetero Lab. Hyderabad. Acetone, methanol, calcium chloride, sodium hydroxide and potassium di-hydrogen phosphate were purchased from E-Merck Limited, Mumbai (India). All the chemicals used were of analytical grade.

Table 1: Formulations of drug loaded microbeads.

\begin{tabular}{|c|c|c|}
\hline $\begin{array}{c}\text { Formulation } \\
\text { Codes }\end{array}$ & Drug (mg) & GG (mg) \\
\hline F1 & 250 & 250 \\
\hline F2 & 250 & 500 \\
\hline F3 & 250 & 750 \\
\hline F4 & Solvent evaporation & Solvent evaporation \\
\hline F5 & $250^{*}$ & 500 \\
\hline F6 & $250^{*}$ & 750 \\
\hline
\end{tabular}

* Microsphere equivalent to $250 \mathrm{mg}$ drug

\section{Methods}

\section{Preparation of D4T loaded calcium-gellan microbeads}

D4T loaded calcium-gellan microbeads were prepared by the method of ionotropic gelation. The formulation codes with quantities are given in Table 1. For formulation F1 to F3, desired quantity of GG was dispersed in double distilled water $(25 \mathrm{ml})$ maintained at $55^{\circ} \mathrm{C}$ to which D4T (250 mg) was added and mixed properly. Finally, the resultant slurry was extruded dropwise into the counterion solution containing a $100 \mathrm{ml}$ mixture of $7.5 \% \mathrm{w} / \mathrm{v}$ calcium chloride using a $10 \mathrm{ml}$ hypodermic syringe (18 G needle) with constant stirring (250 RPM) for $30 \mathrm{~min}$. Then the resulting beads were washed thrice in double distilled water, dried at room temperature for $48 \mathrm{~h}$, and stored in desiccator for further use.

\section{Preparation of D4T loaded ERSPO microspheres ${ }^{8}$ (F4)}

D4T loaded microspheres were prepared by solvent evaporation technique keeping the drug to polymer ratio 1: 1. ERSPO $(1.5 \mathrm{~g})$ was dissolved in $8.5 \mathrm{ml}$ acetone using a magnetic stirrer (Remi Equipments, model 2MIH). D4T (500 mg) and magnesium stearate $(75 \mathrm{mg})$ were dispersed in the polymer solution. The resulting dispersion was then poured into $250 \mathrm{ml}$ beaker, containing the mixture of $100 \mathrm{ml}$ liquid paraffin light and $10 \mathrm{ml}$ $\mathrm{n}$-hexane maintained at the desired temperature, while stirring at $1000 \mathrm{rpm}$. A mechanical stirrer with a blade (4 cm diameter) (Remi Motors, Model No.RO-123R, Mumbai) was used. Stirring was continued for 3 to 5 $\mathrm{h}$ then microspheres were filtered through a Whatman No. 1 filter paper. The residue was washed 4 to 5 times in $50 \mathrm{ml} \mathrm{n}$-Hexane each. Microspheres were dried at room temperature for $24 \mathrm{~h}$. The prepared microspheres were passed through sieve no. \# 60. The fraction that passed through the sieve were collected and taken for embedding within gellan beads.

\section{Preparation of microspheres within microbeads (F5 and F6)}

For preparation of MEM, microspheres equivalent to $250 \mathrm{mg}$ drug was added to slurry of gellan and mixed properly and microbeads were prepared by the method described as in the earlier section. All the formulations were prepared in triplicate to obtain desired quantity and to check repeatability.

\section{Determination of yield, encapsulation efficiency and particle size}

Beads prepared by the above described method were weighed after drying to constant weight and the percent yield was measured using the following formula. 


\begin{tabular}{|c|c|c|c|c|c|c|}
\hline \multicolumn{7}{|c|}{ Table 2: Evaluation parameters for drug loaded microbeads. } \\
\hline $\begin{array}{c}\text { Formulation } \\
\text { codes }\end{array}$ & $\begin{array}{c}\text { Yield } \\
(\mathbf{\%}) \\
(\mathbf{n = 3 )}\end{array}$ & $\begin{array}{c}\text { Carr's Index } \\
(\mathbf{n}=\mathbf{3})\end{array}$ & $\begin{array}{c}\text { MPS } \\
(\boldsymbol{\mu m}) \\
(\mathbf{n}=\mathbf{1 0 0})\end{array}$ & $\begin{array}{c}\text { Drug content } \\
\%\end{array}$ & \% EE & $\mathbf{n}$ value \\
\hline F1 & $94.80 \pm 1.6$ & 5.26 & $474.4 \pm 3.1$ & 1.90 & 3.8 & 0.712 \\
\hline F2 & $90.94 \pm 1.4$ & 8.814 & $498.82 \pm 2.5$ & 3.03 & 9.12 & 0.570 \\
\hline F3 & $92.00 \pm 1.6$ & 4.761 & $530.17 \pm 1.9$ & 2.95 & 11.81 & 0.539 \\
\hline F4 & $85.10 \pm 1.9$ & 7.677 & $218.41 \pm 2.2$ & 47.30 & 94.60 & 0.489 \\
\hline F5 & $87.5 \pm 1.8$ & 6.71 & $544.2 \pm 2.7$ & 22.01 & 66 & 0.979 \\
\hline F6 & $92.15 \pm 1.6$ & 7.381 & $564.2 \pm 2.7$ & 19.20 & 76.8 & 0.947 \\
\hline
\end{tabular}

* MPS: Mean particle size, EE: Encapsulation Efficiency, $n$ : release rate exponent of various formulations by korsmeyer-Peppas model. Each observation is the (mean $\pm \mathrm{SD})$ of three observations.

\section{Percentage Yield $=($ Actual weight $\times 100) /$ Theoretical Weight}

Drug content in the resulting formulations was determined by accurately weighing $100 \mathrm{mg}$ microbeads and triturating it by a mortar and pestle into powder form. The resulting powder was transferred to a $100 \mathrm{ml}$ volumetric flask and the volume was made up to the mark using phosphate buffer $\mathrm{pH}$ 6.8. The resulting mixture was kept shaking on a mechanical shaker for $24 \mathrm{~h}$, after which filtration was carried out using membrane filter $(0.45 \mu \mathrm{m}$ pore size $)$ and after suitable dilution the solution was analysed spectrophotometrically at 266.5 nm using UV - Visible Spectrophotometer (Systronics 2101).

Encapsulation efficiency was calculated by the following formula.

Encapsulation efficiency $=($ Estimated drug content $/$ Theoretical drug content) X 100.

The Particle size of the prepared microbeads was found out by arbitrarily counting average diameter of 100 particles with optical microscope using stage micrometer and eyepiece scale.

\section{Scanning electron microscopy (SEM)}

Scanning electron microscope (Joel JSM -5200) was used to illustrate surface topography of prepared microbeads. The microbeads were placed on a metallic support with a thin adhesive tape and were coated with gold under vacuum (Fine coat, ion sputter JFC -1110) to make them electron conductive. The surface was scanned and photomicrographs were taken by $20 \mathrm{kv}$ accelerating voltage.

\section{Fourier transform infrared spectroscopy (FTIR)}

FTIR spectra were recorded for pure drug, formulation F3 and F6 using FTIR (JASCO 410). Samples were prepared in $\mathrm{KBr}$ disks (2 $\mathrm{mg}$ sample in $200 \mathrm{mg} \mathrm{KBr}$ ). The scanning range was $1000-4500 \mathrm{~cm}^{-1}$ and the resolution was $2 \mathrm{~cm}^{-1}$.

\section{In vitro drug release studies}

The release studies of prepared beads were carried out in the USP-I (basket) dissolution test apparatus (Labinndia-2000, Mumbai, India) using $500 \mathrm{ml}$ of phosphate

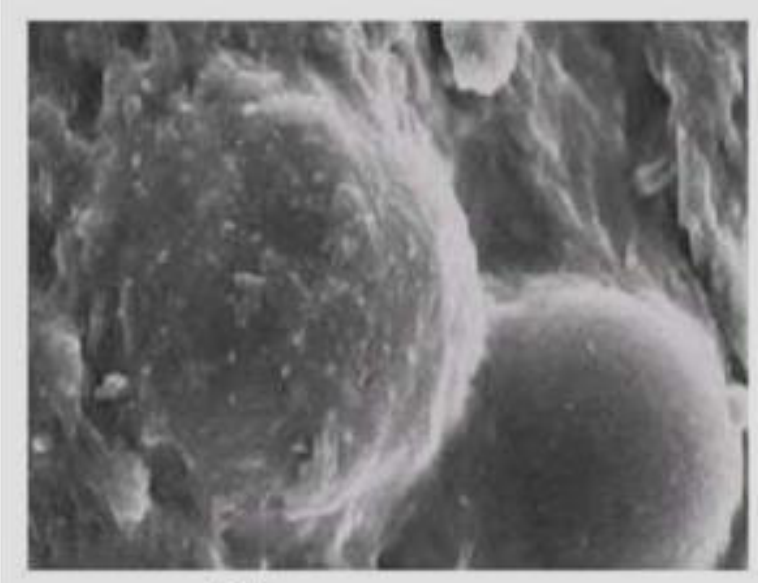

(A)

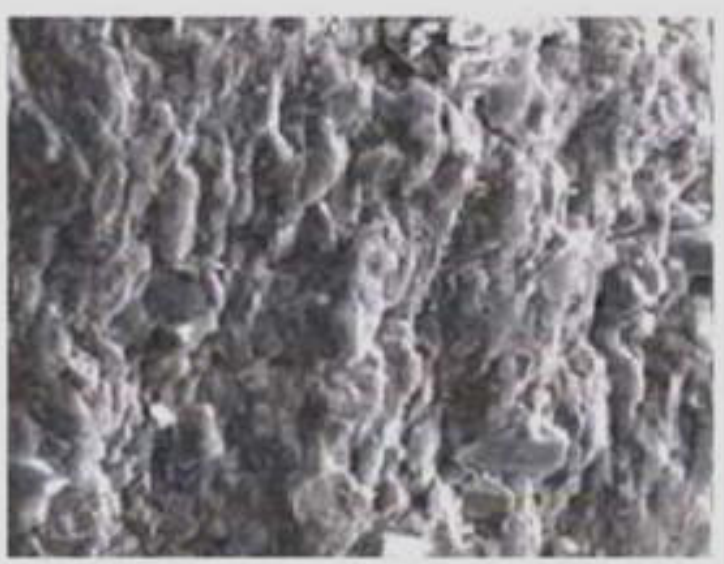

(日)

Figure 1: (A) and (B) (Magnification level 100X) shows the photomicrographs of microspheres of formulation F6 and F3 respectively 

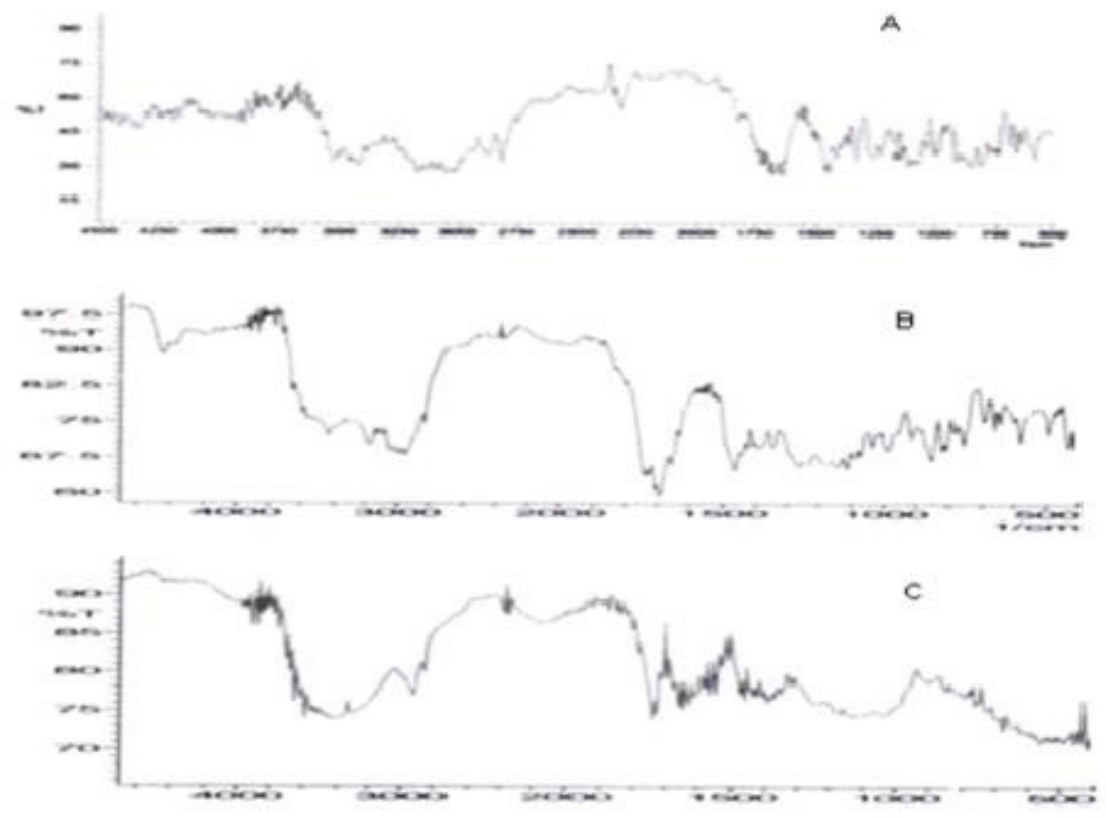

Figure 2: The FTIR spectra of pure drug D4T (A), Formulation F3 (B) and F6 (C).

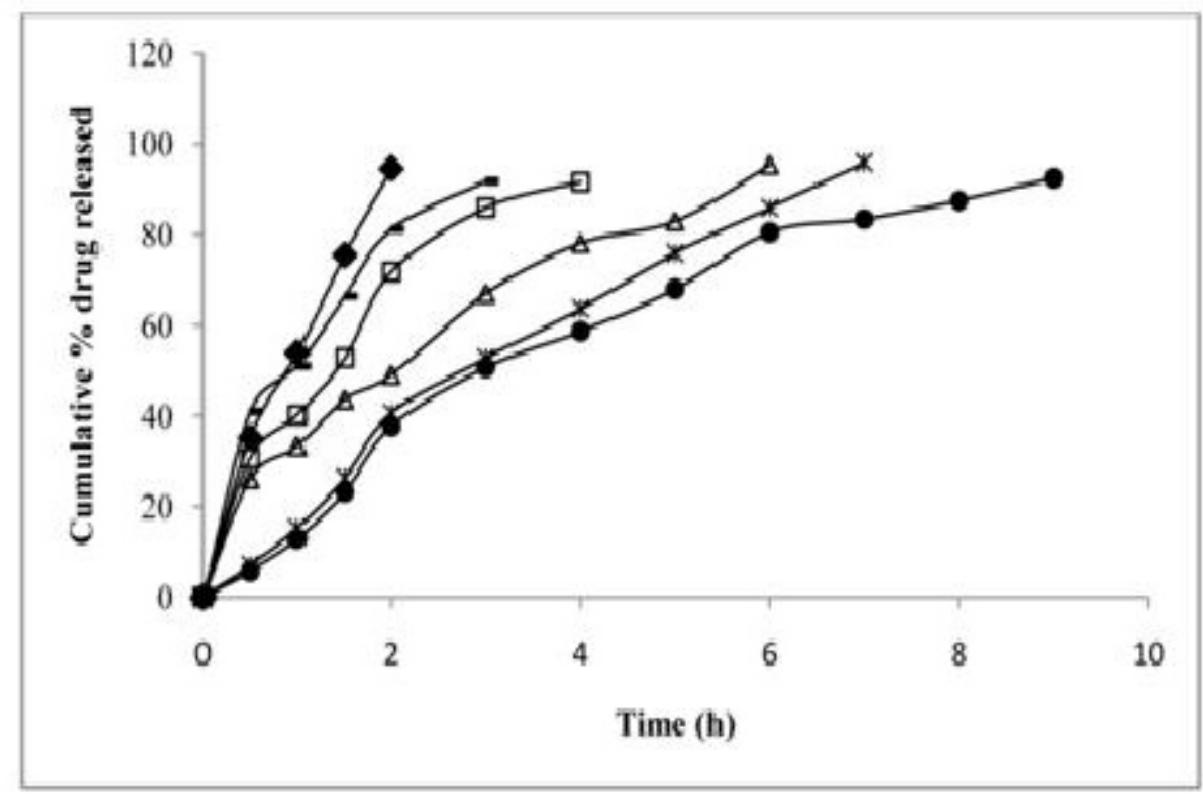

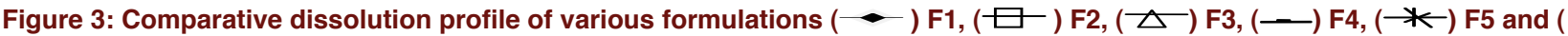
- ) F6. Each observation is the mean \pm S.D. of three observations.

buffer $(6.8 \mathrm{pH})$ maintained at a temperature of $37 \pm$ $0.5^{\circ} \mathrm{C}$ while rotating at $100 \mathrm{RPM}$. Beads equivalent to 50 mg of D4T was taken for dissolution study under sink condition. Samples withdrawn at various time intervals were appropriately diluted and analysed by UV-VIS spectrophotometer (Systronics 2101) at $266.5 \mathrm{~nm}$. Drug release mechanism from beads was determined by using Korsmeyer-Peppas model using the following equation. ${ }^{9}$ $\log M_{t} / M \infty=\log K t^{n}$
Where Mt / $\mathrm{M} \infty=$ fraction of drug released $\mathrm{t}=$ release time

$\mathrm{K}=$ release rate constant

$\mathrm{n}=$ release rate exponent

\section{RESULTS}

Microbeads and microspheres were prepared successfully by the proposed method. The percentage of yield 
in all cases was found to be greater than $87 \%$. All the formulation had Carr's index less than 10. The mean particle size varied from 474 to $564 \mu \mathrm{m}$ (Table 2). The mean particle size and entrapment efficiency varied depending on the concentration of gellan used. The SEM photomicrographs [Figure 1(A)] distinctly shows the microspheres embedded within the microbeads effectively with a smooth surface topography and [Figure 1(B)] shows that the gellan microbeads containing D4T only are spherical in nature with rough surface. The FTIR spectra of pure drug D4T, Formulation F3 and F6 are given in Figure 2. The specific $\mathrm{OH}$ stretching, $\mathrm{NH}$ stretching of secondary amine, $\mathrm{C}-\mathrm{H}$ stretching and $\mathrm{C}=\mathrm{O}$ stretching of pure drug remained unaltered within the formulation $\mathrm{F} 3$ and F6. The rate of release of drug from microbeads was a function of amount of gellan used and in the manner, the drug is incorporated within the formulation (Figure 3). Korsmeyer-Peppas model was utilised to study the release mechanism of the drug from the microbeads. The release rate exponent " $n$ " value of all the formulations lies in the range of 0.48 to 0.94 (Table 2).

\section{DISCUSSION}

Gellan microbeads containing the drug D4T was effectively prepared by using the ionic gelation method using $\mathrm{CaCl}_{2}$ as a crosslinking agent at a concentration of $7.5 \%$.The ERSPO microspheres containing D4T was prepared effectively by the solvent evaporation method. The rotation speed of the stirrer was maintained at an RPM of 1000 in a motto to prepare microspheres with a mean particle size less than $250 \mu \mathrm{m}$ so that it could be effectively passed through a syringe size of $18 \mathrm{G}$ needle. Likewise, the microspheres within microbeads were successfully prepared as described by the above method. The encapsulation efficiency of the formulations F1 to F3 was below $12 \%$. An increase in gellan amount from F1 to F3 increased drug encapsulation efficiency, but it was not as high as we were expecting. Generally the higher the polymer concentration the higher will be the probability of drug surrounded by polymer, which acts as a barrier to prevent diffusion of drug into the external medium, but in this case the higher rate of leaching overcomes the barrier effect of polymer while curing in the $\mathrm{CaCl}_{2}$ solution and hence causes a low entrapment efficiency. The leaching of drug is an osmotic phenomenon. In order to maintain the balance of drug concentration between microbead and curing medium, drug leaches to the medium, which causes low entrapment efficiency. To overcome the above problem syringeable microspheres of D4T was prepared using ERSPO and embedded into microbeads of gellan. ERSPO is generally hydrophobic in nature, which checked the leaching of drug into the external medium while curing of microbeads.

The FTIR study ascertained the compatibility of the drug within the formulation.

The in vitro drug release study clearly indicates the rate of decrease in release of drug is a function of amount of gellan used that is formulation F1 to F3 (Figure 3). This may have resulted due to the fact that at higher concentration of gellan, crosslinking increases, and high-unrestricted volume of matrix, which leads to decrease in the release of the drug. The release rate was found to be more sustained when $\mathrm{D} 4 \mathrm{~T}$ in the form of microsphere was embedded within the formulation F2 and F3 that is formulation F5 and F6 respectively. It is so because if the drug has to release first of all it has to diffuse from the core of the hydrophobic ERSPO microsphere into the gellan matrix and from the gellan matrix to the release medium and hence a more sustaining action. The release pattern of formulation F2 and F3 showed a biphasic nature that is exhibited initial higher percentage of drug release followed by a slower release, but in case of formulation F5 and F6 the release pattern was in a more controlled manner. The drug release mechanism in all formulations excluding F4 was found to be following non Fickian type diffusion with " $n$ " value between 0.5 to 1 (Table 2) whereas in case of formulation $F 4$ the mechanism was Fickian type diffusion.

\section{CONCLUSION}

From the above study, it may be concluded that the therapeutic efficacy of a water-soluble drug can be improved by utilising the novel concept that is microspheres embedded within microbeads for better management of disease.

\section{ACKNOWLEDGEMENT}

The authors are grateful to H.O.D., University Department of Pharmaceutical Sciences, Utkal University, Bhubaneswar, Odisha India, for providing necessary facilities for conducting the research work

\section{CONFLICTS OF INTEREST}

None 


\section{REFERENCES}

1. Benita S. Microencapsulation, Second edition. New York: Taylor \& Francis; 2006.

2. Costa $P$, Jose MS. Modeling and comparison of dissolution profiles. Eur $\mathrm{J}$ Pharm Sci. 2001; 13(2): 123-33.

3. Robinson JR, Lee VH. Controlled Drug Delivery, Second edition. New York: Marcel Dekker; 2005.

4. Sahoo SK, Sahoo SK, Behera A, Patil SV, Panda SK. Formulation, in vitro drug release study and anticancer activity of 5-fluorouracil loaded gellan gum microbeads. Acta Pol Pharm. 2013; 70(1): 123-7.

5. Kang KS, Veeder GT, Mirrasoul PJ, Kaneko T, Cottrell IW. Agar-like polysaccharide produced by a Pseudomonas species: production and basic properties. Appl Environ Microb. 1982; 43(5): 1086-91.
6. O'Neill MA, Selvendran RR, Morris VJ. Structure of the acidic extracellular gelling polysaccharide produced by Pseudomonas elodea. Carbohyd Res.1983; 124(1): 123-33.

7. Sahoo SK, Mallick AA, BarikBB, Senapati PC. Formulation and in vitro Evaluation of Eudragit $₫$ Microspheres of Stavudine. TJPR. 2005; 4(1): 369-757.

8. Sahoo SK, Dhal S, Mohapatro P, Behera BC, Barik BB. Effect of processing temperature on Eudragit RSPO microsphere characteristics in the solvent evaporation process. Pharmazie 2007; 62(8): 638-9.

9. Sahoo SK, Mallick AA, Barik BB, Senapati PC. Preparation and in vitro evaluation of ethyl cellulose microspheres containing Stavudineby the double emulsion method. Pharmazie 2007; 62(2): 117-21. 